ARTICLE

https://doi.org/10.1038/s41467-019-11882-7

\title{
PIF transcription factors link a neighbor threat cue to accelerated reproduction in Arabidopsis
}

Vinicius Costa Galvāo (10 1, Anne-Sophie Fiorucci (1) 1, Martine Trevisan (1) 1, José Manuel Franco-Zorilla², Anupama Goyal ${ }^{1,4}$, Emanuel Schmid-Siegert (10 ${ }^{3}$, Roberto Solano (id ${ }^{2} \&$ Christian Fankhauser (i) $^{1}$

Changes in light quality indicative of competition for this essential resource influence plant growth and developmental transitions; however, little is known about neighbor proximityinduced acceleration of reproduction. Phytochrome B (phyB) senses light cues from plant competitors, ultimately leading to the expression of the floral inducers FLOWERING LOCUS T (FT) and TWIN SISTER of FT (TSF). Here we show that PHYTOCHROME INTERACTING FACTORs 4, 5 and 7 (PIF4, PIF5 and PIF7) mediate neighbor proximity-induced flowering, with PIF7 playing a prominent role. These transcriptional regulators act directly downstream of phyB to promote expression of $F T$ and TSF. Neighbor proximity enhances PIF accumulation towards the end of the day, coinciding with enhanced floral inducer expression. We present evidence supporting direct PIF-regulated TSF expression. The relevance of our findings is illustrated by the prior identification of FT, TSF and PIF4 as loci underlying flowering time regulation in natural conditions.

\footnotetext{
${ }^{1}$ Center for Integrative Genomics, Faculty of Biology and Medicine, University of Lausanne, 1015, Lausanne, Switzerland. ${ }^{2}$ Genomics Unit and Plant Molecular Biology Department, Centro Nacional de Biotecnologia (CSIC), Campus de Cantoblanco, Darwin 3, 28049 Madrid, Spain. ${ }^{3}$ SIB Swiss Institute for Bioinformatics, University of Lausanne, 1015 Lausanne, Switzerland. ${ }^{4}$ Present address: Syngene International Ltd, Bangalore 560 099, India. Correspondence and requests for materials should be addressed to C.F. (email: christian.fankhauser@unil.ch)
} 


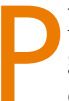
lants depend on sunlight to fuel photosynthesis. Therefore, growing with potentially reduced light availability, as encountered in dense plant communities, constitutes a threat for plant growth and development. Plants perceive potential competitors because of the reflected far-red (FR) light from neighbors, resulting in reduced red (R)/FR ratio (low R/FR), which leads to the conversion of active phytochrome (Pfr) photoreceptors to their inactive Pr form ${ }^{1}$.

In shade-intolerant plants, such as Arabidopsis thaliana, neighbor detection triggers organ elongation to outgrow competitors and precocious flowering ${ }^{1}$. Among the five phytochromes (phys) present in Arabidopsis, phytochrome B (phyB) is the major regulator of shade avoidance ${ }^{1}$. In normal light conditions, photoactive phyB interacts with a class of basic helix-loop-helix (bHLH) transcription factors, the PHYTOCHROMEINTERACTING FACTORS (PIFs), leading to their phosphorylation and degradation via $26 \mathrm{~S}$ proteasome or inactivation through less understood mechanisms ${ }^{2,3}$. In contrast, in low $\mathrm{R} / \mathrm{FR}$, PIF phosphorylation and degradation is prevented due to the reduction in photoactive phyB enabling PIF binding to Gboxes and PBE-boxes in the promoters of many shade-regulated genes $^{3-10}$. We have started to understand how these transcriptional events lead to shade-induced growth responses ${ }^{1}$; however, we know very little about developmental transitions triggered by neighbor proximity.

Accelerated flowering is one of the most dramatic responses in the presence of neighboring plants, particularly for annual plants where it represents a unique and irreversible event. In Arabidopsis, accelerated flowering in low R/FR results from the transcriptional induction of FLOWERING LOCUS T $(F T)$ and its close homolog TWIN SISTER OF FT (TSF) in the phloem companion cells of the leaf vasculature ${ }^{1-14}$. FT acts as a long-distance florigen signal being transported to the shoot apical meristem (SAM) where it forms a flowering activation complex (FAC) after interacting with the basic leucine zipper (bZIP) transcription factor FD and the 14-3-3 proteins ${ }^{15-17}$. At the SAM the FAC induces flowering by activating the expression of flowering time genes, such as the MADS-box SUPPRESSOR OF OVEREXPRESSION OF CO 1 (SOC1) and APETALA1 (AP1) ${ }^{16,17}$.

Despite their importance for shade-induced flowering, the mechanism linking low R/FR ratio perception by phys to enhanced FT and TSF expression in the vasculature remains poorly understood. In Arabidopsis, low R/FR promotes floral transition in a photoperiod-dependent manner ${ }^{18}$ in agreement with the attenuated low R/FR response of the photoperiodic mutant constans $(c o)^{11,18}$. PHYTOCHROME AND FLOWERING TIME 1 (PFT1) was initially proposed to control flowering in response to simulated shade ${ }^{13}$, but was later shown to respond normally to continuous low $\mathrm{R} / \mathrm{FR}^{18,19}$. Here we show that a subset of PIF transcription factors function genetically downstream of phys to regulate flowering time through FT and TSF expression in response to low R/FR.

\section{Results}

PIFs control low R/FR-induced flowering downstream of phyB. PIFs play major roles in neighbor detection growth responses downstream of phyB ${ }^{4,20,21}$. Enhanced PIF expression induces precocious flowering through FT and TSF in the phloem ${ }^{22-24}$. Moreover, plants with impaired HFR1 function, a repressor of PIF activity ${ }^{25}$, display increased $F T$ expression in response to low $\mathrm{R} / \mathrm{FR}^{26}$. Therefore, we hypothesized that PIFs might control flowering time in response to low R/FR. To test this, we scored the flowering transition of PIF loss-of-function mutants under simulated neighbor proximity conditions (Supplementary Fig. 1a, $\mathrm{b}$; hereafter referred to as low R/FR, while high $\mathrm{R} / \mathrm{FR}$ refers to standard condition) and found that PIF7 plays a prominent function to accelerate floral transition under low R/FR (Fig. 1a-c; Supplementary Fig. 2a, b). In addition, mutations in PIF4 and PIF5 further enhanced the pif7 late flowering phenotype, indicating that these genes also contribute to the response (Fig. 1a-c; Supplementary Figs. 2a, b and 3a-c). Importantly, while pif4 pif5 pif7 flowered slightly later than the wild type in high $\mathrm{R} / \mathrm{FR}$, the pif4 pif5 pif7 phenotype was much enhanced in low R/FR (significant interaction between genotype and condition both in terms of days to flowering $(p=0.0007)$ and leaf number $(p=$ $1.52 \mathrm{e}-05)$ ) showing the requirement for those three PIF particularly for accelerated flowering in low R/FR (Fig. 1c; Supplementary Fig. 2b). Moreover, while pif7, pif3 pif4 pif5, and pif4 pif5 pif7 mutants flowered slightly later than the wild type in high R/ FR, in low R/FR late flowering was specifically observed in pif7 and pif4 pif5 pif7 mutants (Fig. 1a-c; Supplementary Fig. 3a-c). Next, we checked whether PIFs mediate precocious flowering of the constitutive shade-avoidance mutant phyB. Consistent with our data in low R/FR, mutations in PIF4, PIF5, and PIF7 were required to fully suppress early flowering in $p h y B$ in high $\mathrm{R} / \mathrm{FR}$ in inductive (long days (LDs)) and non-inductive (short days (SDs)) photoperiods (Fig. 1d, e; Supplementary Fig. 4a-d). Finally, because low R/FR further accelerates flowering in $p h y B$ mutant due to the activity of other phys ${ }^{27,28}$ (Supplementary Fig. 5a-c), we scored phyB pif4 pif5 pif7 flowering in low R/FR. In this condition, phyB pif4 pif5 pif7 flowers later than $p h y B$, at the same time as pif4 pif5 pif7 (Supplementary Fig. 5a-c), further supporting the role of PIF4, PIF5, and PIF7 in the phyB pathway. We conclude that PIF4, PIF5, and PIF7 act genetically downstream of phyB, and possibly other phys, to control low R/FR-induced flowering.

PIFs control FT and TSF expression in response to low R/FR. Because flowering in low R/FR depends on both the growth condition and the genetic background ${ }^{11,18,27}$, we tested the flowering response of $f t, t s f$, $f t$ tsf, and $c o$. In our conditions $f t$ and tsf single mutants responded strongly to low R/FR ${ }^{11,29}$ (Supplementary Fig. 6a-d), whereas $f t t s f$ double mutants presented a reduced low R/FR response, similar to co (Supplementary Fig. $6 \mathrm{a}-\mathrm{d})$, suggesting that FT and TSF together are needed to promote flowering in low R/FR (significant interaction between genotype ( $f t$ vs. $f t$ tsf) and condition both in terms of days to flowering $(p=8.7 \mathrm{e}-05)$ and leaf number $(p<2 \mathrm{e}-16)$ ) (Supplementary Fig. 6d).

To compare the role of FT and TSF in the constitutive shadeavoidance mutant $p h y B$ with plants growing in low $\mathrm{R} / \mathrm{FR}$, we scored the flowering phenotype of phyB ft, phyB tsf, and phyB ft $t s f$. While $p h y B$ tsf double mutants flowered as $p h y B$, both $f t$ and $f t$ tsf abolished $p h y B$ early flowering in standard conditions (Supplementary Fig. 6e). Together, these findings indicate that the $p h y B$ mutant does not phenocopy plants growing in low $\mathrm{R} / \mathrm{FR}$ in terms of flowering, likely because other phys also act in low R/ FR. This is consistent with acceleration of flowering by low R/FR in the phyB mutant ${ }^{27}$ (Supplementary Fig. 5a-c) and the extreme early flowering of $f t$ when combined with higher-order phytochrome mutants 30,31 .

Next, we determined whether PIFs contribute to FT and TSF transcriptional regulation in low R/FR. Transcriptome data ${ }^{32}$ showed that FT messenger RNA (mRNA) levels increased in cotyledons within 90 min after transfer to low R/FR, while such a rapid induction was not observed for TSF (Supplementary Fig. 7a, b). In contrast, this early FT up-regulation was absent in the pif4 pif5 pif7 mutant (Supplementary Fig. 7c). We therefore monitored FT and TSF expression in the wild type and pif4 pif5 pif7 for several days after transfer from high to low R/FR at 


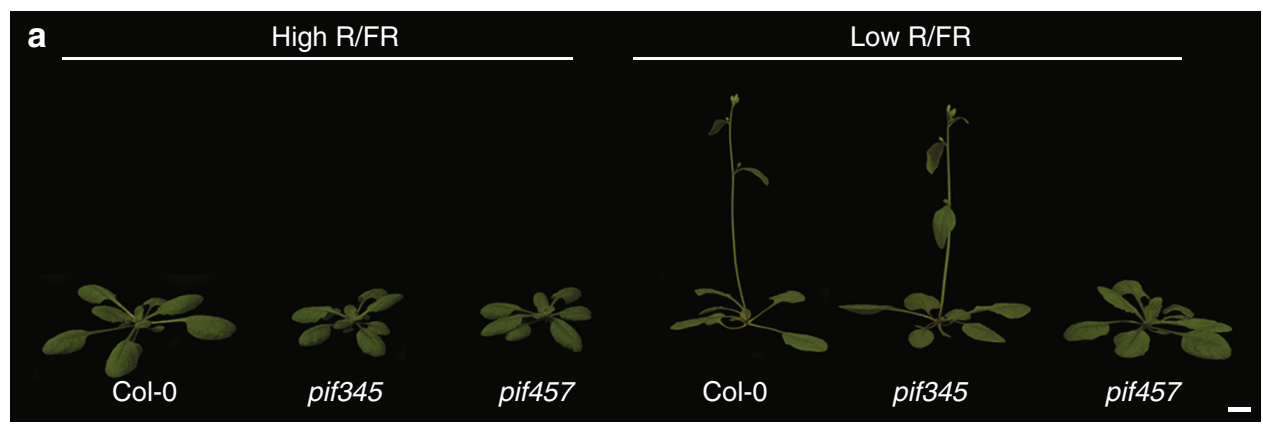

b $\square$ High R/FR $\square$ Low R/FR

C

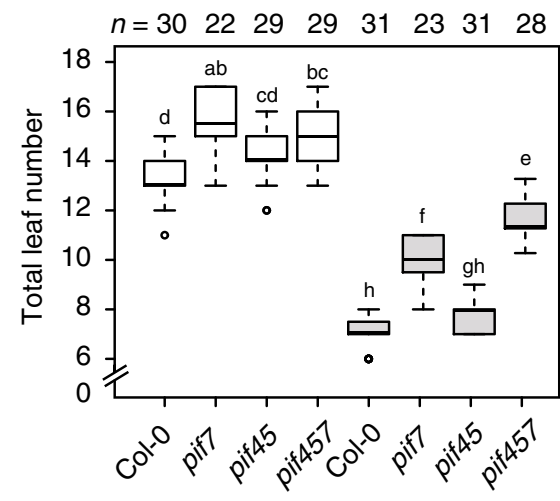

Ratio (high/low R/FR)
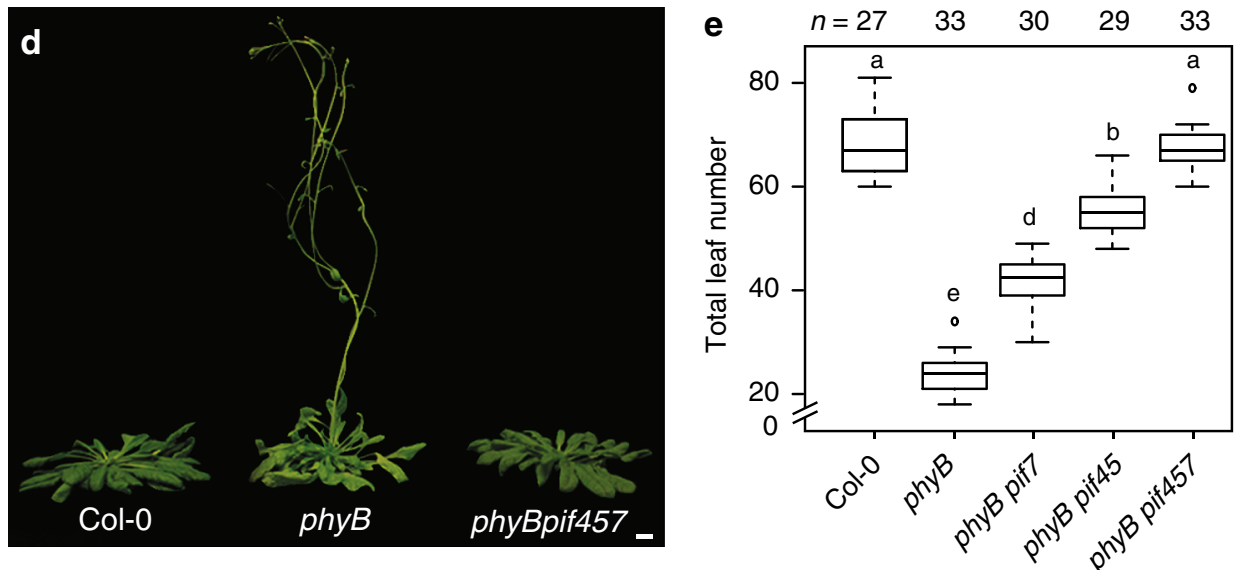

Fig. 1 PHYTOCHROME-INTERACTING FACTORS (PIFs) mediate flowering in the low red/far-red (R/FR) downstream of phytochrome B (phyB). Plants were grown for 5 days in high R/FR for complete de-etiolation and either kept in high R/FR or shifted to low R/FR on day 6 until the onset of flowering. a Twenty-two-day-old Col-0, pif3 pif4 pif5 and pif4 pif5 pif7 grown under long days (LDs) at $22^{\circ} \mathrm{C}$ in high and low R/FR with flowering phenotype represented as total leaf number $\mathbf{b}, \mathbf{c}$ Leaf number ratio (high vs. low R/FR) of plants phenotyped in $\mathbf{a}$. $\mathbf{d}$ Representative image of 53-day-old Col-0, phy $B$ and phyB pif4 pif5 pif7 under short days (SDs) at $22^{\circ} \mathrm{C}$ in high R/FR and flowering phenotype represented as total leaf number $\mathbf{e}$ Boxplots were created using the online BoxPlotR ${ }^{80}$; center line, median; box limits, upper and lower quartiles; whiskers, $1.5 \times$ interquartile range (IQR); dots, outliers. $n$ represents the number of plants phenotyped. Letters represent the significance groups at $p$ value $<0.01$ using one-way analysis of variance (ANOVA), followed by Tukey's honestly significant difference (HSD) test. Scale bar correspond to $1 \mathrm{~cm}$

ZT16, as FT and TSF expression peak at dusk ${ }^{18,33}$. FT and TSF expression were similar in pif4 pif5 pif7 and wild-type plants in high R/FR. In contrast, FT and TSF up-regulation by low R/FR were strongly impaired in pif4 pif5 pif7 (Fig. 2a, b). Consistent with the importance of PIF-dependent TSF up-regulation, ft pif4 pif5 pif7 quadruple mutants flowered later compared to $f t$ and similar to $f t$ tsf under low R/FR (Supplementary Fig. 8a-d). Moreover, in low R/FR TSF expression was reduced in ft pif4 pif5 pif7 compared to the $f t$ single mutant (Supplementary Fig. 8e). Low R/FR-mediated induction of FT and TSF were reduced in pif7 single mutant and further reduced in pif4 pif5 pif7 (Fig. 2c, $\mathrm{d})$, correlating with the flowering time defects of those mutants
(Fig. 1b, c; Supplementary Fig. 2). Similarly, the constitutively higher expression of FT and TSF in $p h y B$ was gradually reduced in higher-order pif mutants (Supplementary Fig. 9a, b). Collectively, these results show that PIF4, PIF5, and PIF7 contribute to low R/FR-mediated floral transition, as well as FT and TSF expression.

In contrast to FT and TSF, CO mRNA expression was only marginally increased by low R/FR light treatments in both wild type and the pif4 pif5 pif7 mutant (Supplementary Fig. 10). In order to determine whether PIF4, PIF5, and PIF7 control low R/ FR-induced flowering exclusively through the CO-FT/TSF pathway, we compared flowering of co and co pif4 pif5 pif7 mutants. 

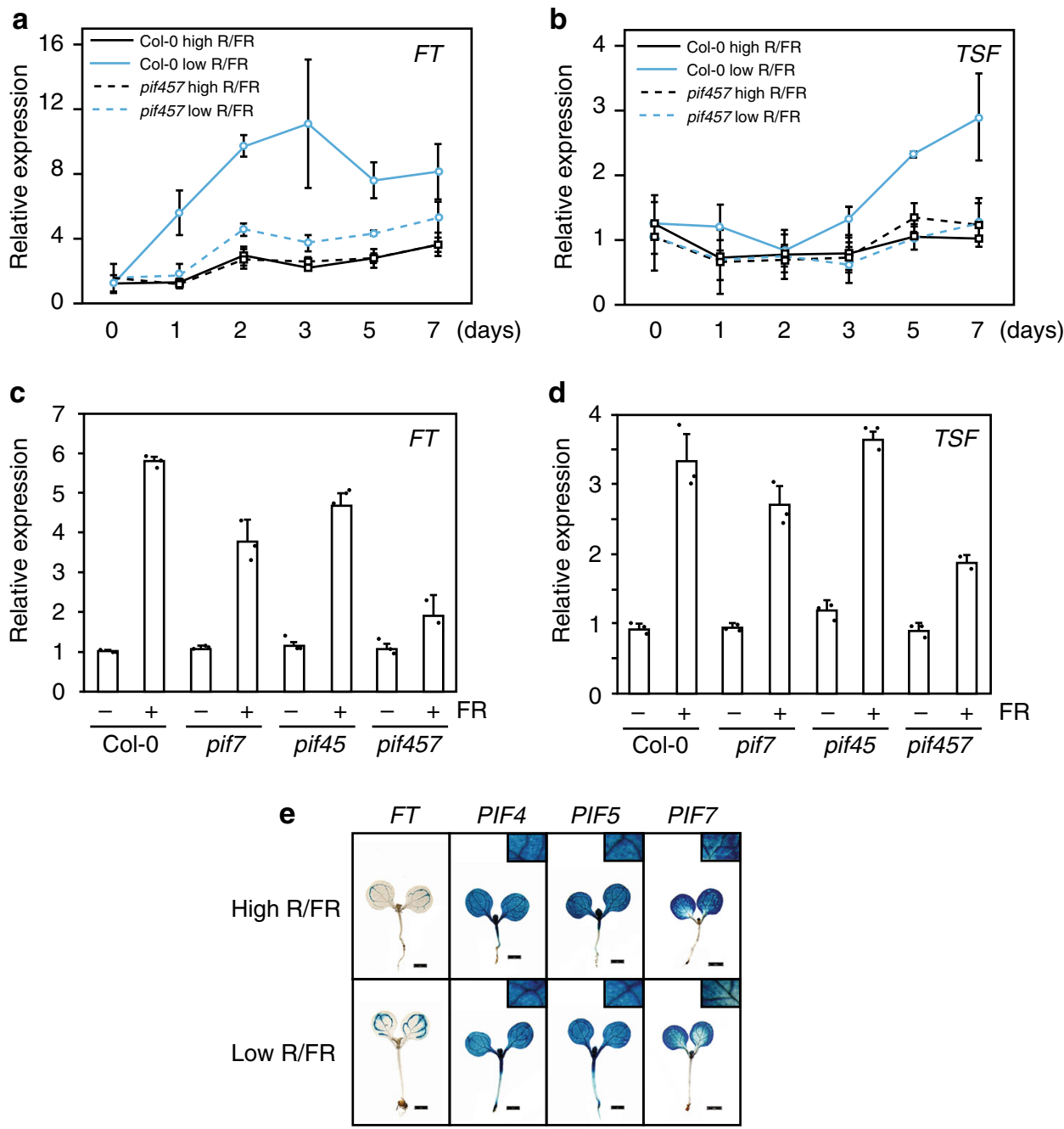

Fig. 2 PHYTOCHROME-INTERACTING FACTORS (PIF) proteins mediate FLOWERING LOCUS (FT) and TWIN SISTER of FT (TFS) expression in the low red/farred (R/FR). a, b FT and TSF messenger RNA (mRNA) level using quantitative real-time PCR (RT-qPCR) after shift from high to low R/FR in Col-0 and pif4 pif5 pif7. Plants were de-etiolated for 5 days under long days (LDs) at $22^{\circ} \mathrm{C}$ in high R/FR and samples were harvested at zeitgeber (ZT) $15-6$ before 0 and $1,2,3,5$, and 7 days after transfer to low R/FR. Error bars represent $2 \times S E M . \mathbf{c}, \mathbf{d} F T$ and TSF mRNA levels of plants growing under LDs at $22^{\circ} \mathrm{C}$ in high and low R/FR. Plants were grown for 5 days in high R/FR for complete de-etiolation. On day 6, plants were either shifted to low R/FR (FR+) or kept in high R/ FR (FR-). Samples were harvested 10 days after sowing at ZT 15-16. Error bars represent standard deviation of three biological and three technical replicates, individual data points are indicated with black dots. e $\beta$-Glucuronidase (GUS) staining of pPIF4::GUS, pPIF5::GUS, and pPIF7::GUS transgenic seedlings 7 days after sowing. Plants were grown on soil for 5 days under LDs at $22^{\circ} \mathrm{C}$ in high R/FR and either kept in the same condition of shifted to low R/FR on day 6. Samples were harvested after GUS staining at ZT 15-16. Scale bar corresponds to $1 \mathrm{~mm}$

Interestingly, the quadruple mutant flowered later than co both in high and low R/FR, indicating that the PIFs also control flowering through a CO-independent pathway (Supplementary Fig. 11a-c). Moreover, similar to $f t$ pif4 pif5 pif7 and $f t t s f$, the quadruple co pif4 pif5 pif7 responded to low R/FR, suggesting that independent flowering pathways mediate low R/FR-induced flowering (Supplementary Figs. $8 \mathrm{a}-\mathrm{c}$ and $11 \mathrm{a}-\mathrm{c}$ ). Collectively, our results identify PIFs as important mediators of FT- and TSF-induced early flowering in response to low R/FR and suggest that PIFs also control flowering through a CO-FT/TSF-independent pathway.

PIF protein accumulation correlates with FT-TSF expression. To better understand how PIFs control FT and TSF expression, we investigated their temporal and spatial expression pattern. Consistent with the vascular expression of FT and TSF during floral transition $^{11,12,34}$ (Fig. 2e), promoter-GUS ( $\beta$-glucuronidase) fusions showed broad PIF4, PIF5, and PIF7 expression, including the leaf vasculature, in seedlings (Fig. 2e). This is consistent with tissue-specific expression analysis of PIF4, PIF5, and PIF7 $7^{35,36}$, indicating that PIF4, PIF5, PIF7, FT, and TSF are expressed in the vasculature. FT mRNA expression in the wild type displayed two strong peaks in response to low R/FR, the first early in the light period and the highest peak around dusk, and a third smaller peak during the night (Fig. 3a) ${ }^{18}$. In contrast, there was no induction of FT expression by low R/FR in pif4 pif5 pif7 (Fig. 3a). The TSF diel expression pattern and its regulation by low R/FR and the PIFs were very similar to FT (Fig. 3a, b). In contrast to FT and TSF, PIF4, PIF5, and PIF7 expression showed one strong peak in the morning as previously observed for PIF4 and PIF5 ${ }^{37}$ (Fig. 3c-e). Low R/FR ratio led to a slower decline from peak levels that were particularly obvious for PIF4 and PIF5, but not significant for the lower amplitude cycling PIF7 gene (Fig. 3c-e). This expression pattern is consistent with a previous study, which showed that low R/FR slows down the circadian clock $^{38}$. Given that phyB inactivation under low R/FR stabilizes PIF4 and PIF5 

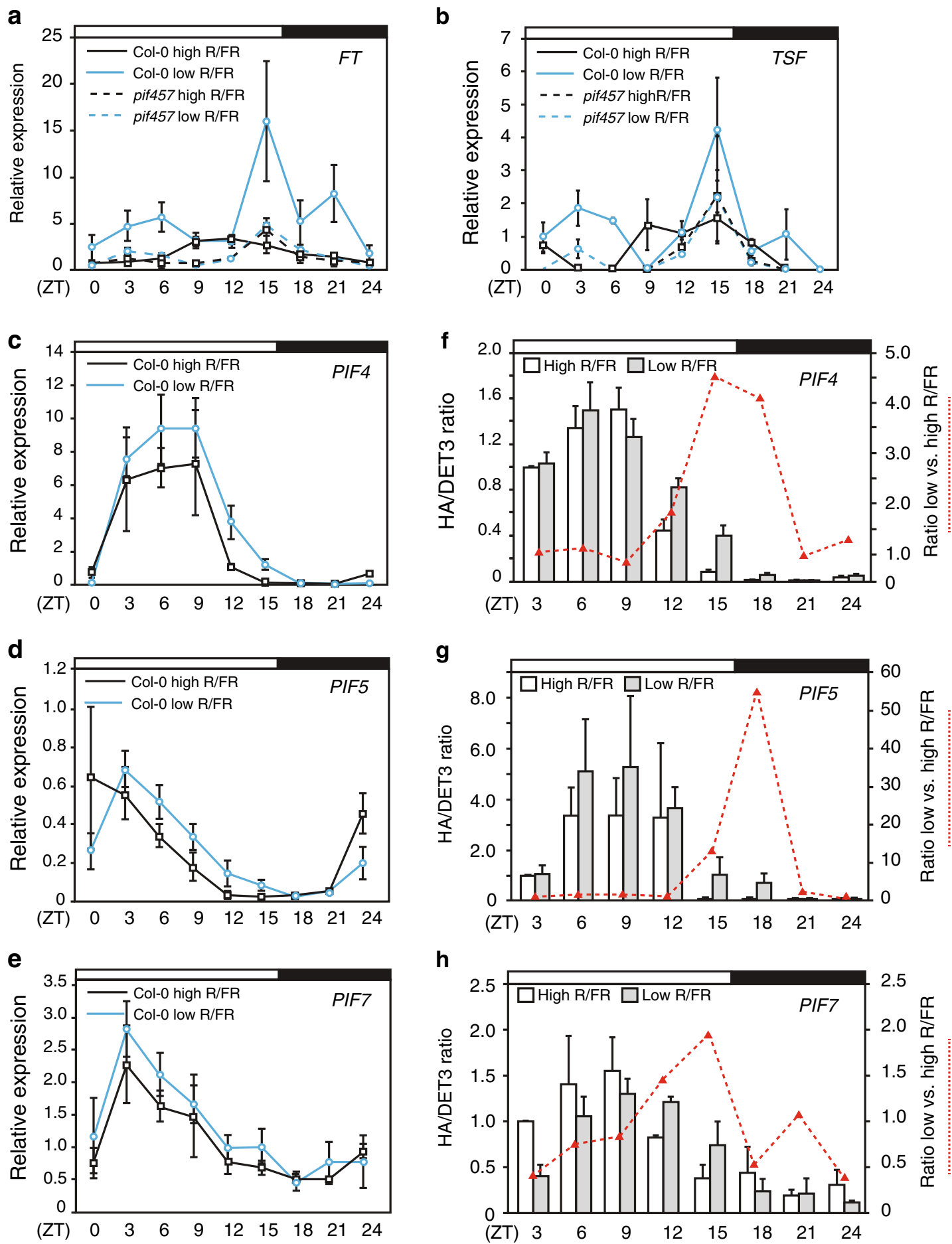

Fig. 3 PHYTOCHROME-INTERACTING FACTORS (PIFs) and FLOWERING LOCUS/TWIN SISTER of FT (FT/TSF) expression pattern in high and low red/farred (R/FR) ratio. Diel time course of FT (a), TSF (b), PIF4 (c), PIF5 (d), and PIF7 (e) over $24 \mathrm{~h}$ harvested every $3 \mathrm{~h}$. Expression levels were determined using quantitative real-time PCR (qRT-PCR) using three biological replicates and three technical replicates of 10-11-day-old Col-0 and pif4pif5pif7 mutant. Plants were grown under high R/FR for 5 days and either kept in the same condition of shifted to low R/FR on day 6 . White and dark bars on top of each chart correspond to light and dark phases, respectively. Error bars correspond to $2 \times$ standard error. Protein accumulation of pif4-101/pPIF4::PIF4-3HA (f), pif5-3/pPIF5:::PIF5-3HA (g), and pif7-2/pPIF7::PIF7-3HA (h) in 10-11-day-old plants harvested every $3 \mathrm{~h}$. White (high R/FR) and gray (low R/FR) bars correspond to the average protein levels of three biological replicates and at least two technical replicates relative to DET3. Red dashed lines represent the PIF protein level ratio of low/high R/FR. Error bars represent standard deviation and white and black bars on top of each chart represent the light and dark phases, respectively 
proteins $^{4}$, we decided to also investigate PIF4, PIF5, and PIF7 protein accumulation. Using genomic HA-tagged lines driven by their own promoters (Supplementary Fig. 12) ${ }^{39}$, we observed diel protein oscillation of HA-tagged PIF4, PIF5, and PIF7 matching mRNA levels (Fig. 3f-h; Supplementary Fig. 13). Moreover, as reported previously ${ }^{20}$, upon transfer to low R/FR we also observed a change in the ratio of slower and faster migrating PIF7 isoforms likely corresponding to different phosphorylation forms (Supplementary Fig. 14). In addition, we noted a transient increase in total PIF7 levels in low R/FR (Supplementary Fig. 14). Interestingly, PIF4 and PIF5 proteins accumulated to higher levels in low R/FR specifically toward the end of the day, correlating with FT and TSF expression (Fig. 3a, b, f, g; Supplementary Fig. 13). There was also a tendency for enhanced end-of-day accumulation of PIF7 in low R/FR, but it was less pronounced compared to PIF4 and PIF5, as also observed for the RNA expression patterns (Fig. 3f-h; Supplementary Fig. 13). However, PIF7 nuclear import is induced by low $\mathrm{R} / \mathrm{FR}^{3}$, indicating that a different mode of low $\mathrm{R} / \mathrm{FR}$ regulation may be involved for PIF7. Multiple levels of PIF regulation by low R/FR is further suggested by the PIF-dependent expression of FT and TSF in low R/FR early in the day, a time at which no significant difference in PIF4, PIF5, or PIF7 accumulation was observed (Fig. 3a, b, f-h).

PIF proteins directly control TSF expression. PIF4 and PIF5 preferentially bind to G-boxes (CACGTG) and PBE-boxes (CATGTG) $)^{8,9}$. Because PIF7 plays a central role in low R/FRinduced flowering and little is known about its DNA-binding preference, we tested its DNA-binding specificity using proteinbinding microarrays. In agreement with recent DNA affinity purification sequencing (DAP-seq) data ${ }^{40}$, and similar to other $\mathrm{PIFs}^{8,9}$, we found that PIF7 binds with high affinity to G-boxes (Fig. 4a). Moreover, as observed for other PIFs, among E-boxes it showed the highest affinity for the PBE-box (Fig. 4a).

Interestingly, the analysis of previously published chromatin immunoprecipitation-sequencing (ChIP-seq) data ${ }^{10}$ revealed a high-confidence PIF4 binding peak containing 1 G-box and 1 PBE-box overlapping with a highly conserved region located 1.5 $\mathrm{kb}$ downstream of FT stop codon (Supplementary Fig. 15) ${ }^{41}$. Because TSF was shown to integrate environmental signals to influence flowering time in natural conditions ${ }^{42}$ and TSF is clearly important for low R/FR-induced flowering in our conditions (Supplementary Fig. 6a-d), we focused our analysis on PIF regulation of TSF expression. We identified no G-boxes, but identified two PBE-boxes in the TSF promoter located 990 and 437 bases upstream of the ATG (Fig. 4b). The analysis of ChIP-seq data ${ }^{10}$ revealed a high-confidence PIF4 peak overlapping with the first PBE-box (-437) (Fig. 4b). To test whether these PBE-boxes are biologically relevant for PIF-mediated TSF expression, we fused its promoter (wild-type and PBE mutants) with luciferase and performed transient transactivation assays in Nicotiana benthamiana. Consistent with PIFs directly regulating TSF expression, PIF4 and PIF7 led to TSF expression (Fig. 4c, d). Importantly, TSF expression was almost completely abolished by a single-nucleotide mutation in the PBE-box present in the PIF4 ChIP peak $(-437)^{10}$ or by mutations in both PBE-boxes and one CAAGTG sequence (Fig. 4c, d). Taken together, our data suggest that PIF4 and PIF7 directly bind to PBE-boxes at TSF promoter to induce its expression.

\section{Discussion}

Neighbor detection leads to precocious flowering that is controlled via the phyB photoreceptor and FT and TSF genes in Arabidopsis $11,13,14,27,29$. In contrast to well-characterized flowering time pathways, such as photoperiod, vernalization, and gibberellic acid, how sensing neighbor proximity cues are linked to expression of "florigen" genes remains poorly understood. Our experiments identify PIF4, PIF5, and PIF7 as transcription factors acting downstream of phyB to induce flowering in response to neighbor proximity through the floral inducers FT and TSF (Figs. 1, 2; Supplementary Figs. 2-5, 8, and 9). On the other hand, both our data and a recent study analyzing flowering and FT expression profiles in more natural conditions reveal a limited role for the PIFs in high $\mathrm{R} / \mathrm{FR}^{43}$ (Figs. 1, 2; Supplementary Figs. 2-4). Therefore, our data reveal an important role for PIFs in regulating flowering particularly in response to low R/FR and identify PIF7 as a novel positive regulator of flowering transition.

The perception and response to low R/FR and elevated temperature share sensing and signaling components, including phyB and PIF $4^{44-46}$. However, while the thermal induction of flowering almost completely depends on FT and is mostly independent of $\mathrm{CO}^{47}$, low R/FR induction of flowering requires $\mathrm{CO}$ (and LDs) and depends both on FT and TSF ${ }^{11,18,29}$ (Supplementary Fig. 6). Under non-inductive warm SDs, a key flowering-inducing function was initially attributed to PIF4 in directly mediating FT expression $^{22}$. However, both the underlying mechanism and the importance of PIF4 in thermal-induced flowering via FT regulation have been questioned $23,24,48,49$. Here, we show that PIF7 plays the most prominent role in low R/FR-induced flowering with additional roles played by PIF4 and PIF5 (Fig. 1; Supplementary Figs. 2 and 3). The flowering phenotype and FT/TSF expression in pif mutants are both consistent with a role for multiple PIFs in low R/FR-induced flowering through the FT/TSF pathway (Figs. 1, 2; Supplementary Figs. 2-5, 8, and 9). Finally, we present evidence for direct regulation of TSF expression by PIF7 and PIF4, providing a mechanistic basis for low R/FRinduced flowering (Fig. 4).

Our genetic data also indicate that additional mechanisms contribute to the regulation of flowering in low R/FR independently of FT-TSF and PIF proteins. Indeed, similar to co and $f t$ tsf (Supplementary Fig. 6) ${ }^{11,18,29}$, co pif4 pif5 pif7 and ft pif4 pif5 pif7 are still responsive to low R/FR (Supplementary Figs. 8 and 11). $C O$ and FT are major flowering time genes expressed in companion cells of leaves and subsequently FT (and possibly TSF) is transported to the SAM to induce flowering ${ }^{17,34}$. Although it could be speculated that low R/FR directly regulate phys and flowering at the SAM, this seems unlikely because it is normally shaded by emerging leaves. Alternatively, other mobile flowering signals, such as gibberellic acid ${ }^{50,51}$, could be produced in leaves downstream of phys ${ }^{52,53}$ and transported to the SAM to induce flowering ${ }^{53-57}$. Indeed, GA metabolism genes are induced by FR light $^{52}$ and the GA-responsive flowering time gene LEAFY (LFY) is up-regulated in the $p h y B$ mutant background ${ }^{53}$. Alternatively, low R/FR could directly regulate the miR156-SPL aging pathway $^{58}$ independently of GA. PIF proteins directly regulate the expression of MIR156 genes and, consequently, several SPL transcription factors in response to end-of-day FR treatments ${ }^{59}$. However, it should be noted that while miR156-SPL age pathway function mostly at the SAM, the tissue-specific expression of PIFs in this tissue have a modest effect on flowering in Arabidopsis ${ }^{23}$. Although we currently do not understand all the pathways induced by low R/FR to accelerate flowering, we identify the phyB-PIF-FT/TSF regulon as one important mechanism (Fig. 4e).

The phyB mutant is often used as a genetic mimic of low R/FRgrown plants. However, when it comes to the regulation of flowering, we and others observe interesting similarities but also differences between both situations. Early flowering in low R/FR and in $p h y B$ requires the activity of several PIFs (Fig. 1; Supplementary Figs. 4 and 5). In contrast, while FT completely accounts for early flowering in $p h y B$ (Supplementary Fig. 6e) both in LD or SD photoperiods, low R/FR triggered flowering requires both FT 


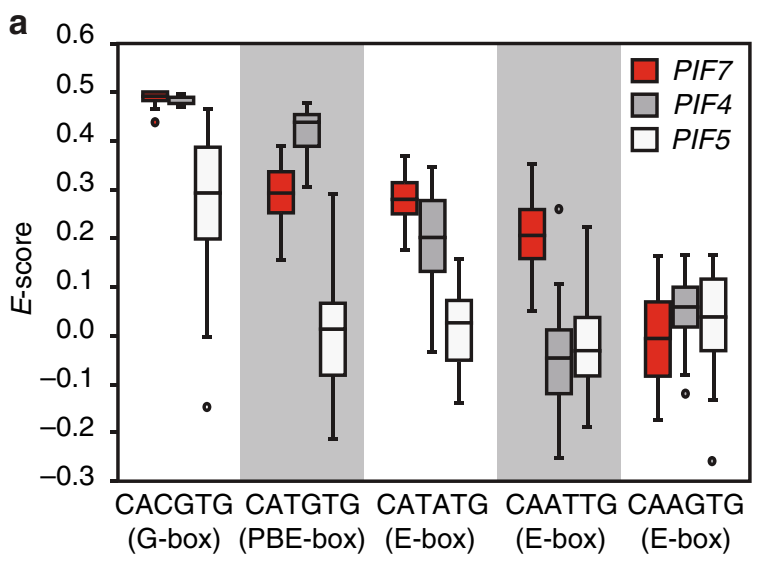

c

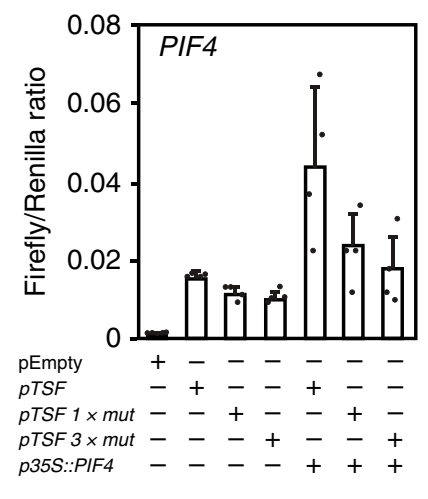

d

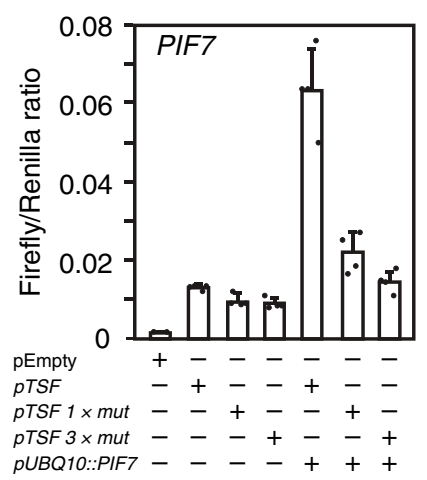

b

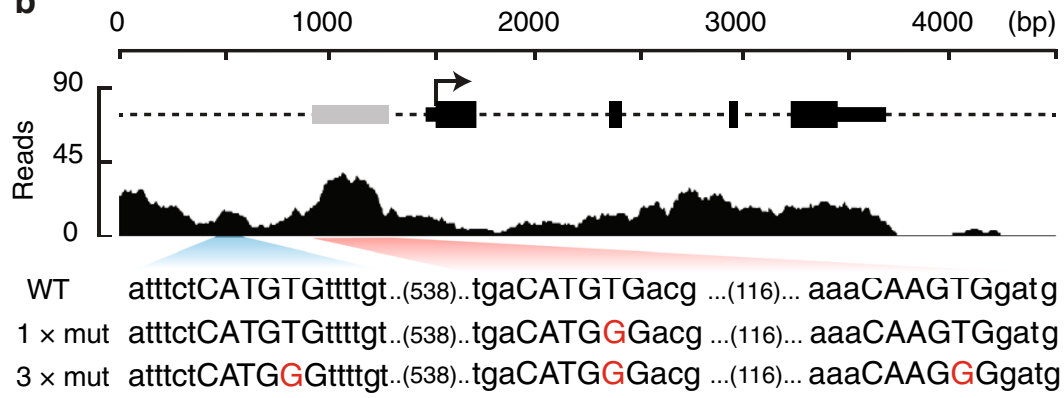

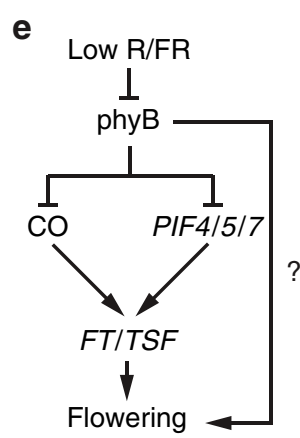

Fig. 4 PHYTOCHROME-INTERACTING FACTORS (PIF) proteins directly regulate TWIN SISTER of FT (TSF) expression. a PIF7 preferentially bind to Gboxes (CACGTG) and PBE-boxes (CATGTG) in protein-binding microarray using PIF7_bHLH-MBP (Supplementary Table 2). Data corresponding to PIF4 and PIF5 were previously described ${ }^{8}$ and shown here for comparison. Box plot represents the distribution of enrichment scores (E-scores) for G- and PBEboxes, as well as for other E-boxes indicated. $E$-score is a rank-based, non-parametric measure of binding affinity that ranges between -0.5 and +0.5 and is calculated from the median intensity of all the oligonucleotide probes that contain a given 8 -mer motif 78 . Boxes represent quartiles $25-75 \%$, the black line within represents the median of the distribution (quartile 50\%) and dots denote outliers of the distribution. b Representation of PIF4 chromatin immunoprecipitation-sequencing (ChIP-seq) reads mapped to the TSF locus ${ }^{10}$. Gray box represents high-confidence PIF4 binding peak at TSF promoter and nucleotide sequence represents the wild-type (WT) and mutant version containing 1 ( $1 \times$ mut) or 3 mutations ( $3 \times$ mut) used for transient dual-luciferase assays in Nicotiana benthamiana. c, d Luciferase ratio corresponds to the average of pTSF::fireflyLUC and p35S:::renillaLUC ratio of four independent infiltrations and error bars correspond to standard deviation; individual data points are indicated with black dots. e Model of low R/FR-regulated flowering time

and TSF and long photoperiods ${ }^{11,18,29,31}$ (Supplementary Fig. 6c). The requirement for inductive photoperiods to trigger low R/FRinduced flowering is also consistent with the reduced low R/FR response of $c o$ and $g i$ mutants ${ }^{18}$. An interesting possibility is that the recently reported interaction of CO with PIF4 ${ }^{24}$, or with other PIFs, could contribute to low R/FR-induced flowering in inductive photoperiods. Indeed, low R/FR leads to increased levels of $\mathrm{CO}^{12}$, PIF4, and PIF5 ${ }^{4,11}$ (Fig. 3; Supplementary Figs. 13 and 14). Moreover, the timing of low R/FR-induced PIF levels is noteworthy as the effect is biggest at the end of the day when $\mathrm{CO}$ protein accumulates and coincides with enhanced FT and TSF expression (Fig. 3). Increased levels of PIF4 and PIF5 proteins at the end of the day could be due to both transcriptional and posttranscriptional effects. Indeed, it was previously shown that low $\mathrm{R} / \mathrm{FR}$ slows down the circadian clock, which may explain the slower decline of PIF4 and PIF5 expression towards the end of the day (Fig. 3c, d) ${ }^{38}$. Therefore, both the higher PIF transcript levels late in the day and enhanced stability of PIF4 and PIF5 protein in low $\mathrm{R} / \mathrm{FR}^{4}$ may contribute to higher PIF levels coinciding with the late FT and TSF expression peaks in low R/FR (Fig. 3). Moreover, we note that low R/FR also leads to higher FT and TSF expression shortly after dawn, a phenomenon that was recently also observed in natural growth conditions (Fig. 3a, b) ${ }^{43}$. Given that at this time of the day we did not observe significant changes in PIF abundance in low vs. high R/FR, we propose that other mechanisms must be operating in shade to control PIF activity. These may include a control of subcellular localization that was reported for $\mathrm{PIF}^{3}$ but also direct inhibition of PIF activity by light-activated phyB, which is more abundant in high $\mathrm{R} / \mathrm{FR}^{46,60-62}$. In summary, the coincidence of high PIF and CO activity towards the end of the day could explain accelerated flowering triggered by neighbor proximity in inductive photoperiods.

In conclusion, multiple mechanisms may contribute to the regulation of PIF protein abundance and activity in low R/FR, but more research is required to fully understand this regulation. However, our data are consistent with the coordinated regulation of FT and TSF expression by PIFs and $\mathrm{CO}$ in response to low $\mathrm{R} / \mathrm{FR}^{24}$ (Figs. 2, 3). These transcriptional events likely operate in the leaf vasculature where $\mathrm{CO}$ is known to regulate FT and TSF expression ${ }^{12,34}$, and the PIFs are also expressed (Fig. 2e) ${ }^{11}$. The mechanism we uncovered here is likely to be significant in natural environments as "florigen" genes FT and TSF, as well as PIF4, were identified as genes underlying regulation of flowering time and shade-avoidance response in nature ${ }^{42,63-65}$. Our study and further deciphering the mechanism(s) regulating neighbor proximity-induced reproduction may also be relevant to increase yields on increasingly restricted agricultural land. 


\section{Methods}

Plant material. All experiments were performed using A. thaliana Columbia (Col0) ecotype. The mutants phyB-966,67, pif4-101 $1^{4}$, pif5- $3^{68}$, pif7- ${ }^{69}$, pif7- $2^{69}, c o-101^{34}$, $f t-10^{70}, t s f-1^{12}$, and the PPIF4::PIF4-3HA line ${ }^{39}$ have been previously characterized. All experiments were performed using pif7-1 allele, except those performed using pif7-2/pPIF7::PIF7-3HA-tPIF7. Mutant lines were confirmed by genotyping using oligonucleotides listed in Supplementary Table 1.

Seeds preparation and growth conditions. Seeds were surface sterilized with $70 \%$ ethanol and stratified for 3 days at $4{ }^{\circ} \mathrm{C}$ in darkness. For all flowering experiments seeds were germinated and seedlings de-etiolated for 5 days in high R/FR ratio (R/ FR 1.20) and on the sixth day low R/FR ratio treatment was started using supplemental FR light (R/FR $\sim 0.20$ ) or plants kept in high R/FR ratio (Supplementary Fig. 1). Low R/FR ratio is achieved using supplemental far-red light from lightemitting diodes light sources. Experiments were performed in plant growth incubator model AR-41L (CLF Plant Climatics) at $~ 22{ }^{\circ} \mathrm{C}$ with $70-80 \%$ humidity and 1:1 of Cool White and Gro-Lux Wide Spectrum fluorescent light (Phillips), with photosynthetically active radiation (PAR) of $200-220 \mu \mathrm{mol} \mathrm{m}^{-2} \mathrm{~s}^{-1}$ (Supplementary Fig. 1). Temperature was monitored using Thermochron iButtons (Maxim Integrated Products) placed at the rosette level and light spectrum and fluence was generated using Ocean Optics USB2000+ spectrometer (Supplementary Fig. 1). LD and SD photoperiod are defined as $16 \mathrm{~h}$ light $/ 8 \mathrm{~h}$ dark and $9 \mathrm{~h}$ light $/ 15 \mathrm{~h}$ dark, respectively.

Flowering time phenotyping and statistical analysis. Flowering time was scored either as total leaf number (rosette and cauline leaves) after bolting or days to flowering after sowing. Statistical analysis of flowering phenotyping was performed using analysis of variance (ANOVA) with post hoc Tukey's honestly significant difference (HSD) test using R software package and statistically significant differences $(p<0.01)$ are represented as different letters. Representative plant images were edited using Adobe Photoshop.

Constructs cloning. The oligonucleotides and constructs described are listed in the Supplementary Table 1. PCR amplifications were performed using Phusion HighFidelity DNA Polymerase (New England Biolabs), unless indicated otherwise. Arabidopsis transformation was performed using Agrobacterium tumefaciens GV3301 strain by floral dip ${ }^{71}$.

PIF4 ( -1 to -2102$)$, PIF5 ( -1 to -2052$)$, and PIF7 ( -1 to -3234$)$ promoter fragments were amplified by PCR using KAPA Taq DNA polymerase (Kapa Biosystems) from MIFL8 and F17J16 BACs, and Col-0 genomic DNA, using the oligos SL-128/SL-129, SL-132/SL-133, and MK-207/MK-208, respectively. PCRamplified fragments were restriction digested and cloned into pCB-308 BamHI sites to create the vectors pAM-01 (pPIF4::GUS) and pAM-03 (pPIF5::GUS), and $B a m \mathrm{HI}$ and $\mathrm{XbaI}$ to create the vector pMK-09 ( $p$ PIF7::GUS). Constructs were transformed in Arabidopsis thaliana Col-0 ecotype.

Genomic PIF7 fragment corresponds to -3185 to +1958 bases relative to the start codon. For cloning PPIF7::PIF7-3HA:tPIF7, genomic fragments were PCR amplified using Phusion polymerase (Thermo Fisher) from genomic DNA using the oligonucleotides oVCG-165/oASF-25 and oVCG-168/oASF-26, and 3HA tag amplified from pCF- $402^{4}$ with the oligonucleotides oVCG-166/oVCG-167. PCR fragments were purified and used for In-Fusion Cloning (Takara) into pKGW vector $^{72}$ previously digested with EcoRV to remove gateway cassette and further linearized with $\mathrm{XbaI}$ to generate the construct pASF-02.

For cloning maltose-binding protein $(M B P)$ expression construct, the sequence corresponding to PIF7 bHLH domain (Supplementary Table 2) was amplified with the oligonucleotides oVCG-226/oVCG-192 from the complementary DNA (cDNA) clone TOPO-U09-H06 and cloned into pMAL-c2 using SacI/XbaI restriction sites to generate the construct pVG-24.

For cloning the reporter construct $P T S F:: f L U C$ the TSF promoter region $(-1$ to $-1551 \mathrm{bp}$ ) was amplified from the BAC clone F9F13 using the oligonucleotides oVCG-440/oVCG-441 and cloned into pGREENII-0800-LUC ${ }^{73}$ XhoI/NotI restriction sites to generate pVG-55. For $p$ TSF 1xmut::fLUC and $p T S F$ 3xmut::fLUC a mutant fragment of the TSF promoter $(-206$ to -1060$)$ was synthesized (Eurofins, Supplementary Table 2) to create the vector pEX-128_pTSF 3xmut. The pTSF 1xmut::fLUC (pVG-84) was generated by restriction digestion of $p E X-$ 128_pTSF 3xmut with PacI/SpeI and cloning the purified fragment into pVG-55 ( $p T S F:: f L U C)$. Full-length $p T S F 3 x m u t:: f L U C$ was created by PCR to amplify partial fragments using the oligos oVCG-374/oVCG-559 and oVCG-560/oVCG-381 from BAC clone F9F13 (pTSF WT), and oVCG-378/oASF-73 from pEX-128_pTSF $3 x m u t$. Finally, the complete $p$ TSF $3 x m u t(-1$ to -1551$)$ was PCR amplified with oVCG-440/oVCG-441 and cloned into pGREENII-0800-LUC XhoI/NotI sites.

RNA isolation and quantitative RT-PCR. Total RNA was extracted using QIAGEN Plant RNeasy kit and cDNA synthesis was carried out using $1 \mu \mathrm{g}$ RNA after an on-column DNAseI digestion (RNAse-Free DNase Set, Qiagen). Reverse transcription (RT) was performed using Superscript II Reverse Transcriptase (Invitrogen, Life Technologies) with random oligonucleotides. Quantitative real-time PCR (qPCR) was performed in three biological replicates with three technical replicates for each sample using the QuantStudio 6 Flex Real-Time PCR System
(Applied Biosystems). Expression data was normalized against UBC and YSL8 reference genes and relative expression analyses estimated Biogazelle qBase software. Gene-specific oligonucleotides used for qPCR reactions and respective efficiencies are listed in Supplementary Table 1.

GUS staining. For GUS staining reactions, samples were fixed with ice-cold $90 \%$ acetone for $30 \mathrm{~min}$ followed by washing twice with $50 \mathrm{mM} \mathrm{NaPO}_{4}$ buffer (pH 7.2). Samples were incubated with staining solution $\left(50 \mathrm{mM} \mathrm{NaPO}_{4}, 0.5 \mathrm{mM}\right.$ potassium ferricyanide, $0.5 \mathrm{mM}$ potassium ferrocyanide, $0.1 \%$ Triton X-100 and $2 \mathrm{mM} \mathrm{X}$ gluc) overnight at $37^{\circ} \mathrm{C}$ in dark. De-staining was performed with ethanol series at room temperature until samples cleared.

Dual-luciferase transient expression in Nicotiana benthamiana. Agrobacterium tumefaciens strain GV3301 was transformed separately with effector and reporter constructs and plated on solid YEP medium supplemented with appropriate antibiotics. After inoculating $30 \mathrm{~mL}$ of YEP medium with $1 \mathrm{~mL}$ of overnight precultures, cells were incubated at $30^{\circ} \mathrm{C}$ with vigorous agitation for $16 \mathrm{~h}$. Nicotiana benthamiana leaves were infiltrated with a mixture of effector (final optical density at wavelength $\left.\left(\mathrm{OD}_{600}\right)=0.3\right)$ and/or reporter (final $\left.\mathrm{OD}_{600}=0.1\right)$ cells along with P19 silencing suppressor (final $\mathrm{OD}_{600}=0.05$ ) using a needleless syringe and infiltrated plants incubated in plant growth room (LD, high R/FR ratio, at $22-23^{\circ}$ C). Three days after incubation, two leaf discs were harvested in liquid nitrogen at zeitgeber (ZT) 8-10 for each biological replicate. After tissue lysis, dual-luciferase assay was performed using Dual-Luciferase Reporter Assay System (Promega). In short, after adding $300 \mu \mathrm{L}$ of $1 \times$ Passive Lysis Buffer, samples were briefly vortexed for $10-15 \mathrm{~s}$ and spinned for $15 \mathrm{~min}$ at $14,000 \times g$ at $4{ }^{\circ} \mathrm{C}$. Ten microliters of supernatant was mixed with $40 \mu \mathrm{L}$ luciferase substrate and chemiluminescence was measured using GloMax 96 Microplate Luminometer (Promega). The second chemiluminescence measurement was performed after adding $25 \mu \mathrm{L}$ of $1 \times$ Stop\&Glo reagent. Relative luciferase activity corresponds to the average firefly/ renilla ratio from four independent infiltrations.

Mapping PIF4 ChIP-seq reads. The original raw data from ref. ${ }^{10}$ obtained from the short read archive:

GSM1665427 ChIP-seq-PIF4_FLAG_Rep1

GSM1665428 ChIP-seq-PIF4_FLAG_Rep2

GSM1665429 ChIP-seq-PIF4_FLAG_Rep3

GSM1665430 ChIP-seq-PIF4_IgG_Rep1

GSM1665431 ChIP-seq-PIF4_IgG_Rep2

Reads were first trimmed using TrimGalore (https://github.com/FelixKrueger/ TrimGalore; v. 0.3.7; -q 30 --length 15) and mapped using bwa (ref. ${ }^{74}$; v 0.7.15) against the TAIR10 reference genome. PCR duplicates were marked using picard tools (http://broadinstitute.github.io/picard; v.2.9.0) and samtools (ref. ${ }^{75}$; v.1.3). The replicate 2 was chosen for the visualization of the peak areas and its mapped reads converted to binary wiggle format using bam2wig (https://github.com/ MikeAxtell/bam2wig) and wigToBigWig (Bio-BigFile-1.01). Processed data, TAIR11 annotation, and published ChIP-seq peaks from ref. ${ }^{10}$ were visualized using Integrative Genome Viewer ${ }^{76}$ and manually edited using Adobe Illustrator.

Western blot analysis. For protein extraction, 15 seedlings were harvested in liquid nitrogen, ground in extraction buffer $(125 \mathrm{mM}$ Tris, $\mathrm{pH} 6.8,4 \%$ sodium dodecyl sulfate (SDS), $20 \%$ glycerol, $0.02 \%$ bromophenolblue, $10 \% \beta$-mercaptoethanol), and heated at $95^{\circ} \mathrm{C}$ for $7 \mathrm{~min}$. After centrifuging for $5 \mathrm{~min}$ at $15,000 \times \mathrm{g}$ at $4{ }^{\circ} \mathrm{C}$, samples were separated using SDS-polyacrylamide gel electrophoresis and transferred to nitrocellulose membrane $0.45 \mu \mathrm{m}$ (Bio-Rad). Blots were probed with anti-HA coupled with horseradish peroxidase (HRP) (1:2000; Roche, cat. $12013819001)$ and polyclonal anti-DET3 $(1: 20,000)$ antibody. HRP-conjugated anti-rabbit immunoglobulins (1:5000, Promega, cat. W4011) were used as secondary antibodies. Chemiluminescence signals were obtained with Immobilon Western Chemiluminescent HRP Substrate (Millipore) on an ImageQuant LAS 4000 mini (GE Healthcare). Relative intensities correspond to the average of HA/ DET of three biological and at least two technical replicates obtained with the software Image Studio Lite. Uncropped Western blots are shown on Supplementary Figs. 16 and 17.

Protein-binding microarray. Protein-binding microarray was performed using Escherichia coli cultures expressing recombinant MBP_PIF7-bHLH proteins (Supplementary Table 2), synthesis of double-stranded microarray, and immunological detection of DNA-protein 77 . In summary, microarrays were scanned in DNA Microarray Scanner (Agilent Technologies) at $2 \mu \mathrm{m}$ resolution and quantified with Feature Extraction 9.0 software (Agilent Technologies). Normalization of probe intensities and calculation of enrichment scores (E-scores) of all the possible 8-mers were carried out with the PBM Analysis Suite ${ }^{78}$. Perl scripts were modified to adapt them to different custom microarray dimensions and Feature Extraction input files ${ }^{79}$. Data of MBP_PIF4-bHLH and MBP-PIF5-bHLH have been previously published and are shown for comparison ${ }^{8}$. 
Statistical analysis. We performed one-way ANOVA (aov) and computed Tukey's HSD (HSD.test) [agricolae package] using the R software.

Reporting summary. Further information on research design is available in the Nature Research Reporting Summary linked to this article.

\section{Data availability}

The authors declare that the data supporting the findings of this study are available within the manuscript and its supplementary files or are available from the corresponding author upon request. Raw data for all experiments is provided in a Source Data file.

Received: 28 October 2018 Accepted: 8 August 2019

Published online: 05 September 2019

\section{References}

1. Fiorucci, A. S. \& Fankhauser, C. Plant strategies for enhancing access to sunlight. Curr. Biol. 27, R931-R940 (2017)

2. Pham, V. N., Kathare, P. K. \& Huq, E. Phytochromes and phytochrome interacting factors. Plant Physiol. 176, 1025-1038 (2018).

3. Huang, X. et al. Shade-induced nuclear localization of PIF7 is regulated by phosphorylation and 14-3-3 proteins in Arabidopsis. Elife https://doi.org/ 10.7554/eLife.31636.001 (2018).

4. Lorrain, S., Allen, T., Duek, P. D., Whitelam, G. C. \& Fankhauser, C. Phytochrome-mediated inhibition of shade avoidance involves degradation of growth-promoting bHLH transcription factors. Plant J. 53, 312-323 (2008).

5. Al-Sady, B., Ni, W., Kircher, S., Schafer, E. \& Quail, P. H. Photoactivated phytochrome induces rapid PIF3 phosphorylation prior to proteasomemediated degradation. Mol. Cell 23, 439-446 (2006).

6. Ni, M., Tepperman, J. M. \& Quail, P. H. Binding of phytochrome B to its nuclear signalling partner PIF3 is reversibly induced by light. Nature $\mathbf{4 0 0}$, 781-784 (1999).

7. Shen, Y., Khanna, R., Carle, C. M. \& Quail, P. H. Phytochrome induces rapid PIF5 phosphorylation and degradation in response to red-light activation. Plant Physiol. 145, 1043-1051 (2007).

8. Hornitschek, P. et al. Phytochrome interacting factors 4 and 5 control seedling growth in changing light conditions by directly controlling auxin signaling. Plant J. 71, 699-711 (2012).

9. Zhang, Y. et al. A quartet of PIF bHLH factors provides a transcriptionally centered signaling hub that regulates seedling morphogenesis through differential expression-patterning of shared target genes in Arabidopsis. PLoS Genet. 9, e1003244 (2013).

10. Pedmale, U. V. et al. Cryptochromes interact directly with PIFs to control plant growth in limiting blue light. Cell 164, 233-245 (2016).

11. Kim, S. Y., Yu, X. \& Michaels, S. D. Regulation of CONSTANS and FLOWERING LOCUS T expression in response to changing light quality. Plant Physiol. 148, 269-279 (2008).

12. Yamaguchi, A., Kobayashi, Y., Goto, K., Abe, M. \& Araki, T. TWIN SISTER OF FT (TSF) acts as a floral pathway integrator redundantly with FT. Plant Cell Physiol. 46, 1175-1189 (2005).

13. Cerdan, P. D. \& Chory, J. Regulation of flowering time by light quality. Nature 423, 881-885 (2003).

14. Halliday, K. J., Salter, M. G., Thingnaes, E. \& Whitelam, G. C. Phytochrome control of flowering is temperature sensitive and correlates with expression of the floral integrator FT. Plant J. 33, 875-885 (2003).

15. Taoka, K. et al. 14-3-3 Proteins act as intracellular receptors for rice Hd3a florigen. Nature 476, $332-\mathrm{U} 397$ (2011)

16. Collani, S., Neumann, M., Yant, L. \& Schmid, M. FT modulates genome-wide DNA-binding of the bZIP transcription factor FD. Plant Physiol. 180, 367-380 (2019).

17. Song, Y. H., Shim, J. S., Kinmonth-Schultz, H. A. \& Imaizumi, T. Photoperiodic flowering: time measurement mechanisms in leaves. Annu. Rev. Plant Biol. 66, 441-464 (2015).

18. Wollenberg, A. C., Strasser, B., Cerdan, P. D. \& Amasino, R. M. Acceleration of flowering during shade avoidance in Arabidopsis alters the balance between FLOWERING LOCUS C-mediated repression and photoperiodic induction of flowering. Plant Physiol. 148, 1681-1694 (2008).

19. Nozue, K. et al. Shade avoidance components and pathways in adult plants revealed by phenotypic profiling. PLoS Genet. 11, e1004953 (2015).

20. Li, L. et al. Linking photoreceptor excitation to changes in plant architecture. Genes Dev. 26, 785-790 (2012).

21. Leivar, P. et al. Dynamic antagonism between phytochromes and PIF family basic helix-loop-helix factors induces selective reciprocal responses to light and shade in a rapidly responsive transcriptional network in Arabidopsis. Plant Cell 24, 1398-1419 (2012).

22. Kumar, S. V. et al. Transcription factor PIF4 controls the thermosensory activation of flowering. Nature 484, 242-245 (2012)

23. Galvão, V. C., Collani, S., Horrer, D. \& Schmid, M. Gibberellic acid signaling is required for ambient temperature-mediated induction of flowering in Arabidopsis thaliana. Plant J. 84, 949-962 (2015).

24. Fernández, V., Takahashi, Y., Gourrierec, J. \& Coupland, G. Photoperiodic and thermosensory pathways interact through CONSTANS to promote flowering at high temperature under short days. Plant J. 86, 426-440 (2016).

25. Hornitschek, P., Lorrain, S., Zoete, V., Michielin, O. \& Fankhauser, C. Inhibition of the shade avoidance response by formation of non-DNA binding bHLH heterodimers. EMBO J. 28, 3893-3902 (2009).

26. Sessa, G. et al. A dynamic balance between gene activation and repression regulates the shade avoidance response in Arabidopsis. Genes Dev. 19, 2811-2815 (2005)

27. Halliday, K. J., Koornneef, M. \& Whitelam, G. C. Phytochrome B and at least one other phytochrome mediate the accelerated flowering response of Arabidopsis thaliana L. to low red/far-red ratio. Plant Physiol. 104, 1311-1315 (1994).

28. Devlin, P. F. et al. Phytochrome D acts in the shade-avoidance syndrome in Arabidopsis by controlling elongation growth and flowering time. Plant Physiol. 119, 909-915 (1999).

29. King, R. W., Hisamatsu, T., Goldschmidt, E. E. \& Blundell, C. The nature of floral signals in Arabidopsis. I. Photosynthesis and a far-red photoresponse independently regulate flowering by increasing expression of FLOWERING LOCUS T (FT). J. Exp. Bot. 59, 3811-3820 (2008).

30. Strasser, B., Sanchez-Lamas, M., Yanovsky, M. J., Casal, J. J. \& Cerdan, P. D. Arabidopsis thaliana life without phytochromes. Proc. Natl. Acad. Sci. USA 107, 4776-4781 (2010).

31. Inigo, S., Alvarez, M. J., Strasser, B., Califano, A. \& Cerdan, P. D. PFT1 the MED25 subunit of the plant Mediator complex, promotes flowering through CONSTANS dependent and independent mechanisms in Arabidopsis. Plant J. 69, 601-612 (2012)

32. Kohnen, M. V. et al. Neighbor detection induces organ-specific transcriptomes, revealing patterns underlying hypocotyl-specific growth. Plant Cell 28, 2889-2904 (2016).

33. Jang, S., Torti, S. \& Coupland, G. Genetic and spatial interactions between FT, TSF and SVP during the early stages of floral induction in Arabidopsis. Plant J. 60, 614-625 (2009)

34. Takada, S. \& Goto, K. Terminal flower2, an Arabidopsis homolog of heterochromatin protein 1, counteracts the activation of flowering locus $\mathrm{T}$ by constans in the vascular tissues of leaves to regulate flowering time. Plant Cell 15, 2856-2865 (2003).

35. Kim, S. et al. Auxin contributes to the intraorgan regulation of gene expression in response to shade. Plant Physiol. 177, 847-862 (2018).

36. You, Y. et al. Phloem companion cell-specific transcriptomic and epigenomic analyses identify MRF1, a regulator of flowering. Plant Cell 31, 325-345 (2019).

37. Nozue, K. et al. Rhythmic growth explained by coincidence between internal and external cues. Nature 448, 358-U311 (2007).

38. Jiménez-Gómez, J. M., Wallace, A. D. \& Maloof, J. Network analysis identifies ELF3 as a QTL for the shade avoidance response in Arabidopsis. PLoS Genet. 6, e1001100 (2010).

39. Zhang, B. et al. BLADE-ON-PETIOLE proteins act in an E3 ubiquitin ligase complex to regulate PHYTOCHROME INTERACTING FACTOR 4 abundance. Elife 6, https://doi.org/10.7554/eLife.26759 (2017).

40. O’Malley, R. C. et al. Cistrome and epicistrome features shape the regulatory DNA landscape. Cell 166, 1598 (2016).

41. Zicola, J., Liu, L., Tanzler, P. \& Turck, F. Targeted DNA methylation represses two enhancers of FLOWERING LOCUS T in Arabidopsis thaliana. Nat. Plants 5, 300-307 (2019).

42. Frachon, L. et al. Intermediate degrees of synergistic pleiotropy drive adaptive evolution in ecological time. Nat. Ecol. Evol. 1, 1551-1561 (2017).

43. Song, Y. H. et al. Molecular basis of flowering under natural long-day conditions in Arabidopsis. Nat. Plants 4, 824-835 (2018).

44. Legris, M. et al. Phytochrome B integrates light and temperature signals in Arabidopsis. Science 354, 897-900 (2016).

45. Koini, M. A. et al. High temperature-mediated adaptations in plant architecture require the bHLH transcription factor PIF4. Curr. Biol. 19, 408-413 (2009)

46. Jung, J. H. et al. Phytochromes function as thermosensors in Arabidopsis. Science 354, 886-889 (2016).

47. Balasubramanian, S., Sureshkumar, S., Lempe, J. \& Weigel, D. Potent induction of Arabidopsis thaliana flowering by elevated growth temperature. PLoS Genet. 2, e106 (2006) 
48. Sureshkumar, S., Dent, C., Seleznev, A., Tasset, C. \& Balasubramanian, S. Nonsense-mediated mRNA decay modulates FLM-dependent thermosensory flowering response in Arabidopsis. Nat. Plants 2, 16055 (2016).

49. Seaton, D. D. et al. Linked circadian outputs control elongation growth and flowering in response to photoperiod and temperature. Mol. Syst. Biol. 11, 776 (2015).

50. Eriksson, S., Bohlenius, H., Moritz, T. \& Nilsson, O. GA4 is the active gibberellin in the regulation of LEAFY transcription and Arabidopsis floral initiation. Plant Cell 18, 2172-2181 (2006).

51. Tal, I. et al. The Arabidopsis NPF3 protein is a GA transporter. Nat. Commun. 7, 11486 (2016).

52. Hisamatsu, T., King, R. W., Helliwell, C. A. \& Koshioka, M. The involvement of gibberellin 20-oxidase genes in phytochrome-regulated petiole elongation of Arabidopsis. Plant Physiol. 138, 1106-1116 (2005).

53. Blazquez, M. A. \& Weigel, D. Independent regulation of flowering by phytochrome B and gibberellins in Arabidopsis. Plant Physiol. 120, 1025-1032 (1999).

54. Yu, S. et al. Gibberellin regulates the Arabidopsis floral transition through miR156-targeted SQUAMOSA PROMOTER BINDING-LIKE transcription factors. Plant Cell 24, 3320-3332 (2012).

55. Galvao, V. C., Horrer, D., Kuttner, F. \& Schmid, M. Spatial control of flowering by DELLA proteins in Arabidopsis thaliana. Development 139, 4072-4082 (2012).

56. Porri, A., Torti, S., Romera-Branchat, M. \& Coupland, G. Spatially distinct regulatory roles for gibberellins in the promotion of flowering of Arabidopsis under long photoperiods. Development 139, 2198-2209 (2012).

57. Hyun, Y. et al. Multi-layered regulation of SPL15 and cooperation with SOC1 integrate endogenous flowering pathways at the Arabidopsis shoot meristem. Dev. Cell 37, 254-266 (2016).

58. Wang, J. W., Czech, B. \& Weigel, D. miR156-regulated SPL transcription factors define an endogenous flowering pathway in Arabidopsis thaliana. Cell 138, 738-749 (2009).

59. Xie, Y. et al. Phytochrome-interacting factors directly suppress MIR156 expression to enhance shade-avoidance syndrome in Arabidopsis. Nat. Commun. 8, 348 (2017).

60. Park, E. et al. Phytochrome B inhibits binding of phytochrome-interacting factors to their target promoters. Plant J. 72, 537-546 (2012).

61. Park, E., Kim, Y. \& Choi, G. Phytochrome B requires PIF degradation and sequestration to induce light responses across a wide range of light conditions. Plant Cell 30, 1277-1292 (2018).

62. Qiu, Y. et al. Mechanism of early light signaling by the carboxy-terminal output module of Arabidopsis phytochrome B. Nat. Commun. 8, 1905 (2017).

63. Brock, M. T., Maloof, J. N. \& Weinig, C. Genes underlying quantitative variation in ecologically important traits: PIF4 (phytochrome interacting factor 4) is associated with variation in internode length, flowering time, and fruit set in Arabidopsis thaliana. Mol. Ecol. 19, 1187-1199 (2010).

64. Brachi, B. et al. Linkage and association mapping of Arabidopsis thaliana flowering time in nature. PLoS Genet. 6, e1000940 (2010).

65. Brachi, B., Faure, N., Bergelson, J., Cuguen, J. \& Roux, F. Genome-wide association mapping of flowering time in Arabidopsis thaliana in nature: genetics for underlying components and reaction norms across two successive years. Acta Bot. Gall. 160, 205-219 (2013).

66. Neff, M. M., Neff, J. D., Chory, J. \& Pepper, A. E. dCAPS, a simple technique for the genetic analysis of single nucleotide polymorphisms: experimental applications in Arabidopsis thaliana genetics. Plant J. 14, 387-392 (1998).

67. Reed, J. W., Nagpal, P., Poole, D. S., Furuya, M. \& Chory, J. Mutations in the gene for the red/far-red light receptor phytochrome $\mathrm{B}$ alter cell elongation and physiological responses throughout Arabidopsis development. Plant Cell 5, 147-157 (1993).

68. Fujimori, T., Yamashino, T., Kato, T. \& Mizuno, T. Circadian-controlled basic/helix-loop-helix factor, PIL6, implicated in light-signal transduction in Arabidopsis thaliana. Plant Cell Physiol. 45, 1078-1086 (2004).

69. Leivar, P. et al. The Arabidopsis phytochrome-interacting factor PIF7, together with PIF3 and PIF4, regulates responses to prolonged red light by modulating phyB levels. Plant Cell 20, 337-352 (2008).

70. Yoo, S. K. et al. CONSTANS activates SUPPRESSOR OF OVEREXPRESSION OF CONSTANS 1 through FLOWERING LOCUS T to promote flowering in Arabidopsis. Plant Physiol. 139, 770-778 (2005).

71. Clough, S. J. \& Bent, A. F. Floral dip: a simplified method for Agrobacterium-mediated transformation of Arabidopsis thaliana. Plant J. 16, 735-743 (1998).
72. Karimi, M., Inze, D. \& Depicker, A. GATEWAY vectors for Agrobacteriummediated plant transformation. Trends Plant Sci. 7, 193-195 (2002).

73. Hellens, R. P. et al. Transient expression vectors for functional genomics, quantification of promoter activity and RNA silencing in plants. Plant Methods 1, 13 (2005)

74. Li, H. \& Durbin, R. Fast and accurate short read alignment with Burrows-Wheeler transform. Bioinformatics 25, 1754-1760 (2009).

75. $\mathrm{Li}, \mathrm{H}$. et al. The Sequence alignment/map (SAM) format and SAMtools. Bioinformatics 25, 2078-2079 (2009).

76. Robinson, J. T. et al. Integrative genomics viewer. Nat. Biotechnol. 29, 24-26 (2011).

77. Godoy, M. et al. Improved protein-binding microarrays for the identification of DNA-binding specificities of transcription factors. Plant J. 66, 700-711 (2011).

78. Berger, M. F. \& Bulyk, M. L. Universal protein-binding microarrays for the comprehensive characterization of the DNA-binding specificities of transcription factors. Nat. Protoc. 4, 393-411 (2009).

79. Franco-Zorrilla, J. M. et al. DNA-binding specificities of plant transcription factors and their potential to define target genes. Proc. Natl. Acad. Sci. USA 111, 2367-2372 (2014).

80. Krzywinski, M. \& Altman, N. Visualizing samples with box plots. Nat. Methods 11, 119-120 (2014).

\section{Acknowledgements}

We thank Koji Goto for $p F T:: G U S$ line and co-101 seeds, Andrea Maran, Séverine Lorrain, and Markus Kohnen for genomic pPIF5::PIF5-3HA and pPIF4/5/7::GUS lines, Prof. Markus Schmid for $f t-10$ mutant, Prof. Hongtao Liu for $p$ GREENII-0800 vector, Dr Rodrigo S. Reis for assistance with dual-luciferase assays, Genomic Technologies Facility (GTF) for assistance with RT-qPCR assays, Frédéric Schütz (SIB) for statistical advice, Dr. Adriana Arongaus, Dr. Martina Legris, and Dr. Olivier Michaud for critical reading of the manuscript. Work in the Fankhauser lab is funded by the University of Lausanne and grants from the Swiss National Science Foundation (no. 310030B_179558 and CRSII3_154438). V.C.G. was supported by EMBO long-term fellowship (ALTF 2932013).

\section{Author contributions}

Experimental design by V.C.G. and C.F. Experimental work and data analysis by V.C.G., A.-S.F., M.T., A.G., J.M.F.-Z, E.S.-S. and R.S. Wrote the manuscript V.C.G. and C.F.

\section{Additional information}

Supplementary Information accompanies this paper at https://doi.org/10.1038/s41467019-11882-7.

Competing interests: The authors declare no competing interests.

Reprints and permission information is available online at http://npg.nature.com/ reprintsandpermissions/

Peer review information: Nature Communications thanks Keara Franklin and the other, anonymous, reviewer(s) for their contribution to the peer review of this work. Peer reviewer reports are available.

Publisher's note: Springer Nature remains neutral with regard to jurisdictional claims in published maps and institutional affiliations.

Open Access This article is licensed under a Creative Commons Attribution 4.0 International License, which permits use, sharing, adaptation, distribution and reproduction in any medium or format, as long as you give appropriate credit to the original author(s) and the source, provide a link to the Creative Commons license, and indicate if changes were made. The images or other third party material in this article are included in the article's Creative Commons license, unless indicated otherwise in a credit line to the material. If material is not included in the article's Creative Commons license and your intended use is not permitted by statutory regulation or exceeds the permitted use, you will need to obtain permission directly from the copyright holder. To view a copy of this license, visit http://creativecommons.org/licenses/by/4.0/.

(C) The Author(s) 2019 